PROCEEDINGS OF THE

AMERICAN MATHEMATICAL SOCIETY

Volume 125, Number 7, July 1997, Pages 1997-2005

S 0002-9939(97)04119-1

\title{
SOLUTION CURVES FOR SEMILINEAR EQUATIONS ON A BALL
}

\author{
PHILIP KORMAN
}

(Communicated by Jeffrey B. Rauch)

\begin{abstract}
We show that the set of positive solutions of semilinear Dirichlet problem on a ball of radius $R$ in $R^{n}$

$$
\Delta u+\lambda f(u)=0 \text { for }|x|<R, u=0 \text { on }|x|=R
$$

consists of smooth curves. Our results can be applied to compute the direction of bifurcation. We also give an easy proof of a uniqueness theorem due to Smoller and Wasserman (1984).
\end{abstract}

\section{INTRODUCTION}

We consider positive solutions of a semilinear problem

$$
\Delta u+\lambda f(u)=0 \text { for }|x|<R, u=0 \text { on }|x|=R
$$

on a ball of radius $R$ in $R^{n}$. Our study of (1.1) will depend in a crucial way on the properties of the linearized equation

$$
\Delta w+\lambda f^{\prime}(u) w=0 \text { in }|x|<R, w=0 \text { on }|x|=R .
$$

By a well-known result of B. Gidas, W.-M. Ni and L. Nirenberg [5] positive solutions of (1.1) are radially symmetric, i.e. $u=u(r)$, where $r=|x|$, and moreover, $u^{\prime}(r)<0$ for all $r \in(0, R)$. By a theorem of C.S. Lin and W.-M. Ni [10] all solutions of (1.2) are also radially symmetric. Accordingly, we shall study the ODE versions of these equations; see the equations (2.1) and (2.2) below.

We show that solutions of (1.1) lie on smooth curves in the $(\lambda, u)$ "plane", i.e. either solution of (1.1) can be continued in $\lambda$, or else it is a simple turning point. Apart from smoothness, our only assumption on $f(u)$ is that $f(0) \geq 0$. We distinguish two possibilities. If (1.2) admits only the trivial solution (one then refers to the solution $u$ of (1.1) as non-degenerate) then the implicit function theorem applies, allowing us to continue solutions. If $u$ is degenerate, i.e. (1.2) admits nontrivial solutions, then we show that the bifurcation theorem of M.G. Crandall and P.H. Rabinowitz [1] applies. Our Lemma 2.1 not only verifies the crucial condition of that theorem, but allows in some cases the computation of the direction of bifurcation. In fact, in a recent paper of P. Korman, Y. Li and T. Ouyang [9]

Received by the editors January 9, 1996.

1991 Mathematics Subject Classification. Primary 35J60.

Key words and phrases. Dirichlet problem on a ball, Crandall-Rabinowitz theorem.

(C)1997 American Mathematical Society 
a two-dimensional variant of this lemma was used to prove an exact multiplicity result for a class of equations which includes the problem

$$
\Delta u+\lambda u(u-a)(b-u)=0 \text { in }|x|<R, u=0 \text { on }|x|=R,
$$

in two dimensions, with constant $0<a<\frac{1}{2} b$. Namely, we proved that there is a critical $\lambda_{0}>0$, so that the problem has exactly zero, one or two positive solutions, depending on whether $\lambda$ is less than, equal to or greater than $\lambda_{0}$.

We also give a simple proof of a uniqueness result of J.A. Smoller and A.G. Wasserman [12]. In [12] it is proved that the problem (1.1) under the conditions (2.13) and (2.14) can have at most one positive solution. The proof is rather complicated. Then in the last section of [12] it is shown that under the same conditions any positive solution of (1.1) is non-degenerate. We found it advantageous to reverse the steps. First, we give a very simple proof of non-degeneracy of solutions. This implies that any solution curve cannot turn. Uniqueness of solutions will follow, if one can show that all solutions of (1.1) lie on a single solution curve. This we do by showing that there is only one curve approaching "infinity", obtaining a considerably simpler proof of uniqueness. This approach to uniqueness, which does not involve the "shooting" method, is quite general. In a forthcoming paper we obtain a similar uniqueness result for a wide class of nonlinearities on annular domains.

Without loss of generality we shall assume that $R=1$. We shall denote the derivative of $u(r)$ by either $u^{\prime}$ or $u_{r}$, and will mix both notations to make proofs more transparent.

Next we state a bifurcation theorem of Crandall-Rabinowitz [1].

Theorem 1.1 ([1]). Let $X$ and $Y$ be Banach spaces. Let $(\bar{\lambda}, \bar{x}) \in \boldsymbol{R} \times X$ and let $F$ be a continuously differentiable mapping of an open neighborhood of $(\bar{\lambda}, \bar{x})$ into $Y$. Let the null-space $N\left(F_{x}(\bar{\lambda}, \bar{x})\right)=\operatorname{span}\left\{x_{0}\right\}$ be one-dimensional and $\operatorname{codim} R\left(F_{x}(\bar{\lambda}, \bar{x})\right)=1$. Let $F_{\lambda}(\bar{\lambda}, \bar{x}) \notin R\left(F_{x}(\bar{\lambda}, \bar{x})\right)$. If $Z$ is a complement of $\operatorname{span}\left\{x_{0}\right\}$ in $X$, then the solutions of $F(\lambda, x)=F(\bar{\lambda}, \bar{x})$ near $(\bar{\lambda}, \bar{x})$ form a curve

$$
(\lambda(s), x(s))=\left(\bar{\lambda}+\tau(s), \bar{x}+s x_{0}+z(s)\right),
$$

where $s \rightarrow(\tau(s), z(s)) \in \boldsymbol{R} \times Z$ is a continuously differentiable function near $s=0$ and $\tau(0)=\tau^{\prime}(0)=0, z(0)=z^{\prime}(0)=0$.

\section{Global solution curves on a ball}

In this section we consider positive solutions of the problem

$$
u^{\prime \prime}(r)+\frac{n-1}{r} u^{\prime}(r)+f(u)=0, \quad r \in(0,1), \quad u^{\prime}(0)=u(1)=0 .
$$

We shall need the linearized equation

$$
w^{\prime \prime}+\frac{n-1}{r} w^{\prime}+f^{\prime}(u) w=0, \quad r \in(0,1), \quad w^{\prime}(0)=w(1)=0 .
$$

The following lemma generalizes a similar result for the $n=2$ case in [7].

Lemma 2.1. Let $f(u) \in C^{2}\left(\bar{R}_{+}\right)$satisfy $f(0) \geq 0$. Assume that (2.2) admits a nontrivial solution. Then

$$
\int_{0}^{1} f(u) w r^{n-1} d r>0
$$


Proof. Notice that condition $f(0) \geq 0$ and the Hopf's boundary lemma imply that (see e.g. [3] $) u^{\prime}(1)<0$. We may also assume that $w^{\prime}(1)<0$. Differentiate $(2.1)$ :

$$
u^{\prime \prime \prime}+\left(\frac{n-1}{r} u^{\prime}\right)^{\prime}+f^{\prime}(u) u^{\prime}=0 .
$$

Multiply the equation (2.2) by $r^{n} u^{\prime}$, and subtract from it (2.4) multiplied by $r^{n} w$, then integrate over $(0,1)$. Obtain

$$
\int_{0}^{1}\left[r^{n} u^{\prime} w^{\prime \prime}-r^{n} u^{\prime \prime \prime} w+(n-1) r^{n-1} u^{\prime} w^{\prime}-r^{n}\left(\frac{n-1}{r} u^{\prime}\right)^{\prime} w\right] d x=0 .
$$

Integrate by parts in the first, second and fourth terms. Obtain:

$$
\begin{gathered}
\left.r^{n} u^{\prime} w^{\prime}\right|_{0} ^{1}+\int_{0}^{1}\left[-n r^{n-1} u^{\prime} w^{\prime}-r^{n} u^{\prime \prime} w^{\prime}+n r^{n-1} w u^{\prime \prime}+r^{n} w^{\prime} u^{\prime \prime}\right. \\
\left.+(n-1) r^{n-1} u^{\prime} w^{\prime}+n(n-1) r^{n-2} u^{\prime} w+(n-1) r^{n-1} u^{\prime} w^{\prime}\right] d r=0 .
\end{gathered}
$$

After cancellations

$$
u^{\prime}(1) w^{\prime}(1)+\int_{0}^{1}\left[(n-2) r^{n-1} u^{\prime} w^{\prime}+n r^{n-1}\left(u^{\prime \prime}+\frac{n-1}{r} u^{\prime}\right) w\right] d r=0 .
$$

Integrating by parts again,

$$
\begin{aligned}
\int_{0}^{1} r^{n-1} u^{\prime} w^{\prime} d r & =-\int_{0}^{1}\left[(n-1) r^{n-2} u^{\prime} w+r^{n-1} u^{\prime \prime} w\right] d r \\
& =-\int_{0}^{1} r^{n-1}\left(u^{\prime \prime}+\frac{n-1}{r} u^{\prime}\right) w d r .
\end{aligned}
$$

Using this in (2.6)

$$
u^{\prime}(1) w^{\prime}(1)+2 \int_{0}^{1} r^{n-1}\left(u^{\prime \prime}+\frac{n-1}{r} u^{\prime}\right) w d r=0 .
$$

Finally, using the equation (2.1), we conclude

$$
\int_{0}^{1} r^{n-1} f(u) w d r=\frac{1}{2} u^{\prime}(1) w^{\prime}(1)>0 .
$$

We now present a theorem on local parameterization of positive solutions, which is similar to Theorem 4.1 in [6]. We denote by $B$ the unit ball in $R^{n}$.

Theorem 2.1. Assume $f(u) \in C^{2}(\bar{R}+)$ satisfies $f(0) \geq 0$. Let $\left(\lambda_{0}, u_{0}\right) \in R \times$ $C^{2, \alpha}(\bar{B})$ be any solution of

$$
\Delta u+\lambda f(u)=0 \text { for }|x|<1, u=0 \text { for }|x|=1,
$$

where $u(0)=p_{0}>0$. Then there are a neighborhood $U$ of $\left(\lambda_{0}, u_{0}\right)$ in $R \times C^{2, \alpha}(\bar{B})$ and a $C^{1}$ curve

$$
C=\left\{(\lambda(p), u(p)), \quad p \in\left(p_{0}-\delta, p_{0}+\delta\right) \text { for some } \delta>0\right\}
$$

of solutions of $(2.7)$ with $\left(\lambda\left(p_{0}\right), u\left(p_{0}\right)\right)=\left(\lambda_{0}, p_{0}\right)$, such that all solutions of $(2.7)$ in $U$ are on $C$. The parameter $p$ is the maximum of solution, $p=u(0)$. 
Proof. We will show first that solutions of (2.7) lie on a smooth curve near $\left(\lambda_{0}, u_{0}\right)$. We consider the linearization of (2.7)

$$
\Delta w+\lambda_{0} f^{\prime}\left(u_{0}\right) w=0 \text { in } B, w=0 \text { on } \partial B .
$$

If (2.8) has only the trivial solution, then the implicit function theorem applies, producing a $C^{1}$ curve of solutions near $\left(\lambda_{0}, u_{0}\right)$. So assume that $(2.8)$ has nontrivial solutions at $\left(\lambda_{0}, u_{0}\right)$. We shall verify the conditions of the Crandall-Rabinowitz theorem at that point. We denote $X=\left\{u \in C^{2, \alpha}(\bar{B}) \mid u=0\right.$ on $\left.\partial B\right\}$ and $Y=$ $C^{\alpha}(\bar{B})$, and consider the map $F: R_{+} \times X \rightarrow Y$ defined by $F(\lambda, u)=\Delta u+\lambda f(u)$. Clearly zeros of the map $F$ are solutions of (2.7), and the null-space of $F_{u}\left(\lambda_{0}, u_{0}\right)$ consists of solutions of (2.8). We recall a result of C.S. Lin and W.-M. Ni [10] which states that any solution of (2.8) is radially symmetric. It follows that the null-space of $F_{u}\left(\lambda_{0}, u_{0}\right)$ is one-dimensional, since it can be parameterized by $w^{\prime}(1)$ (using a uniqueness theorem from ODE's). Since $F_{u}\left(\lambda_{0}, u_{0}\right)$ is a Fredholm operator of index zero, it follows that codim $R\left(F_{u}\left(\lambda_{0}, u_{0}\right)\right)=1$. Finally, if the condition $F_{\lambda}\left(\lambda_{0}, u_{0}\right) \notin R\left(F_{u}\left(\lambda_{0}, u_{0}\right)\right)$ was violated, one could find $z \in X$ satisfying

$$
\Delta z+\lambda_{0} f^{\prime}\left(u_{0}\right) z=f\left(u_{0}\right) \text { in } B, z=0 \text { on } \partial B .
$$

Since $w$ is solution of the homogeneous version of (2.9), we have by the Fredholm alternative

$$
0=\int_{B} f(u) w d x=n \omega_{n} \int_{0}^{1} f(u) w r^{n-1} d r
$$

contradicting Lemma 2.1. We conclude that the Crandall-Rabinowitz theorem applies, which implies that the solution set of $(2.7)$ near $\left(\lambda_{0}, u_{0}\right)$ consists of a $C^{1}$ curve C.

It remains to show that the curve $C$ can be parameterized by the amplitude $u(0)=p$. By Theorem 1.1, in the case when $F_{u}\left(\lambda_{0}, u_{0}\right)$ is non-invertible, we can represent the curve $C$ near $\left(\lambda_{0}, u_{0}\right)$ as

$$
u(r, s)=u_{0}(r) \pm s w(r)+o(s) \text { for } s \text { close to } 0,
$$

where $w=w(r)$ is a non-trivial solution of the linearized equation (2.8). Hence we can locally parameterize the solution by $p=u(0)$, provided $w(0) \neq 0$. If we assume the contrary, then $w(r)$ solves

$$
w^{\prime \prime}+\frac{n-1}{r} w^{\prime}+\lambda f^{\prime}(u) w=0, \quad w(0)=w^{\prime}(0)=0 .
$$

An obvious modification of the argument in L.A. Peletier and J. Serrin [11, p.190] shows that $w(r)$ vanishes identically, a contradiction. Next we consider the case when $F_{u}\left(\lambda_{0}, u_{0}\right)$ is invertible. Then the implicit function theorem applies, and for small $\Delta \lambda$

$$
u\left(r, \lambda_{0}+\Delta \lambda\right)=u\left(r, \lambda_{0}\right)+u_{\lambda}\left(r, \lambda_{0}\right) \Delta \lambda+o(\Delta \lambda) .
$$

As before we can parameterize solutions locally by $p=u(0)$, provided $u_{\lambda}(0) \neq 0$. If we assume the contrary, then $u_{\lambda}$ satisfies

$$
\begin{aligned}
u_{\lambda}^{\prime \prime}(r)+\frac{n-1}{r} u_{\lambda}^{\prime}(r) & +\lambda f^{\prime}(u) u_{\lambda}(r)=-f(u) \text { for } r \in(0,1), \\
u_{\lambda}(0) & =u_{\lambda}^{\prime}(0)=u_{\lambda}(1)=0 .
\end{aligned}
$$


Proceeding similarly to [6], we notice that $z(r) \equiv r u_{r}$ is a solution of

$$
\begin{aligned}
z^{\prime \prime}(r)+\frac{n-1}{r} z^{\prime}(r) & +\lambda_{0} f^{\prime}\left(u_{0}\right) z(r)=-2 \lambda_{0} f\left(u_{0}\right), \\
z(0) & =z^{\prime}(0)=0 .
\end{aligned}
$$

From (2.11) and (2.12) we see that $w(r) \equiv 2 \lambda_{0} u_{\lambda}-z(r)$ is a solution of (2.10). Hence, $w \equiv 0$ on $[0,1]$; in particular $w(1)=0$, i.e. $u^{\prime}(1)=0$, a contradiction.

Remark. After this paper was submitted, we learned from E.N. Dancer that Theorem 2.1 can also be proved using the methods of his paper [2]. Our Lemma 2.1 has other applications, including computation of the direction of bifurcation, see e.g. $[9]$.

Next we turn to a uniqueness result for (1.1) first proved by J.A. Smoller and A.G. Wasserman [12]. We assume that $f(u) \in C^{2}\left(R_{+}\right)$and it satisfies

$$
\begin{gathered}
f^{\prime}(u)>\frac{f(u)}{u} \text { for almost all } u>0, \\
f^{\prime \prime}(u) \leq 0 \text { for } u>0 .
\end{gathered}
$$

Our first goal is to show that under these conditions the linearized equation (2.2) has only the trivial solution. This will follow from the following lemmas.

Recall that we had excluded the possibility that $w(0)=0$, so that we may assume for definiteness that $w(0)>0$.

Lemma 2.2. Assume that condition (2.13) holds. Then any solution of the linearized equation (2.2) must vanish somewhere on $(0,1)$.

Proof. Assume that on the contrary $w(r)>0$ on $(0,1)$. From the equations $(2.1)$ and (2.2) we obtain

$$
\left(u^{\prime} w-u w^{\prime}\right)^{\prime}+\frac{n-1}{r}\left(u^{\prime} w-u w^{\prime}\right)+\left(f(u)-f^{\prime}(u) u\right) w=0 .
$$

Letting $q(r) \equiv u^{\prime} w-u w^{\prime}$, we obtain using $(2.13)$

$$
\left(r^{n-1} q\right)^{\prime}>0
$$

Integrating over $(0,1)$ we obtain

$$
q(1)>0 .
$$

This contradicts the obvious equality $q(1)=0$.

Lemma 2.3. Assume $w(0)>0$, and let $\xi$ be the first (smallest) point where $w(\xi)=0$. Then

$$
f(u(\xi)) \leq 0 .
$$

Proof. We differentiate both equation (2.1) and (2.2), obtaining

$$
\begin{gathered}
u_{r}^{\prime \prime}+\frac{n-1}{r} u_{r}^{\prime}+f^{\prime}(u) u_{r}-\frac{n-1}{r^{2}} u_{r}=0, \\
w_{r}^{\prime \prime}+\frac{n-1}{r} w_{r}^{\prime}+f^{\prime}(u) w_{r}-\frac{n-1}{r^{2}} w_{r}+f^{\prime \prime}(u) u_{r} w=0 .
\end{gathered}
$$


We now multiply the equation (2.17) by $r^{n-1} w^{\prime}$ and subtract from it the equation (2.18) multiplied by $r^{n-1} u^{\prime}$,

$$
\begin{array}{r}
r^{n-1}\left(u^{\prime \prime} w^{\prime}-u^{\prime} w^{\prime \prime}\right)^{\prime}+(n-1) r^{n-2}\left(u^{\prime \prime} w^{\prime}-u^{\prime} w^{\prime \prime}\right) \\
=r^{n-1} f^{\prime \prime}(u) u_{r}^{2} w .
\end{array}
$$

Setting $p(r) \equiv u^{\prime \prime} w^{\prime}-u^{\prime} w^{\prime \prime}$, and using our condition (2.14), we obtain from (2.19)

$$
\frac{d}{d r}\left[r^{n-1} p\right] \leq 0
$$

Integrating over $(0, \xi)$, we conclude

$$
p(\xi) \leq 0 .
$$

Since $w(\xi)=0$, we express from $(2.2) w^{\prime \prime}(\xi)=-\frac{n-1}{\xi} w^{\prime}(\xi)$, and then by $(2.20)$ and $(2.1)$

$$
0 \geq p(\xi)=\left(u^{\prime \prime}(\xi)+\frac{n-1}{\xi} u^{\prime}(\xi)\right) w^{\prime}(\xi)=-f(u(\xi)) w^{\prime}(\xi) .
$$

Since clearly $w^{\prime}(\xi)<0$, we conclude that $f(u(\xi)) \leq 0$.

Next we need an elementary lemma.

Lemma 2.4. Let the function $f(u)$ satisfy (2.13) and (2.14). Then $f(u)$ has the following properties: $f(0)<0, f(u)$ changes sign at most once on $(0, \infty)$. If moreover we are given that the problem (2.1) has a positive solution, then $f(u)$ changes sign exactly once on $(0, \infty)$, and it is an increasing function on $(0, \infty)$. Finally, there is a constant $c>0$, such that

$$
\lim _{u \rightarrow \infty} \frac{f(u)}{u}=c .
$$

Proof. Condition (2.13) implies that $\left(\frac{f(u)}{u}\right)^{\prime}>0$, i.e. the function $\frac{f(u)}{u}$ is increasing, and then $\frac{f(u)}{u}>\frac{f(\delta)}{\delta}$ for all $u>\delta>0$. Letting $\delta \rightarrow 0$, we rule out the possibility of $f(0)>0$. If one assumes $f(0)=0$, then $\frac{f(u)}{u}>\lim _{u \rightarrow 0} \frac{f(u)}{u}=f^{\prime}(0)$. I.e., $f(u)>f^{\prime}(0) u$ for all $u>0$. On the other hand, by (2.14) $f^{\prime}(u) \leq f^{\prime}(0)$ for all $u>0$. Integrating, we get $f(u) \leq f^{\prime}(0) u$, a contradiction. Hence $f(0)<0$. By (2.13) $f(u)$ cannot change sign more than once. If $f(u)$ is negative for all $u>0$ then by the maximum principle (2.1) cannot have positive solutions. Since we are interested in positive solutions of (2.1) we shall assume from now on that $f(u)$ changes sign exactly once, say at $u=\theta$.

For $u>\theta$ the function $f^{\prime}(u)$ is positive and decreasing. Hence $\lim _{u \rightarrow \infty} f^{\prime}(u)$ exists, call it $c \geq 0$. The possibility of $c=0$ is inconsistent with $\frac{f(u)}{u}$ being increasing. Hence $c>0$, and (2.21) follows by L'Hóspital rule.

Remark. The class of functions satisfying the conditions (2.13) and (2.14) includes functions like $f(u)=c_{1} u-c_{2}-e^{-\alpha u}$ with positive $c_{1}, c_{2}$ and $\alpha$.

Theorem 2.2. Under the conditions (2.13) and (2.14) the problem (2.2) admits only the trivial solution. (In other words, any solution of (2.1) is non-degenerate.)

Proof. Assume that $w(r)$ is a nontrivial solution of (2.2). Let $\xi$ be its smallest root, whose existence follows by Lemma 2.2. Since $u(r)$ is a decreasing function, it follows, in view of Lemma 2.3, that

$$
f(u(r))<0 \text { for all } r \in(\xi, 1) .
$$


We may assume that $w(r)$ is positive near $r=1$. We can then find $\eta \in(\xi, 1)$ such that $w(\eta)>0, w^{\prime}(\eta)=0$ and

$$
w^{\prime}(r) \leq 0 \text { for } r \in(\eta, 1) .
$$

(E.g. $\eta$ the largest point of local maximum of $w(r)$ on $(\xi, 1)$.) We now multiply the equation (2.1) by $w^{\prime}$, the equation $(2.2)$ by $u^{\prime}$ and add, obtaining

$$
\frac{d}{d r}\left(u^{\prime} w^{\prime}\right)+\frac{2}{r}(n-1) u^{\prime} w^{\prime}+\frac{d}{d r}(f(u) w)=0 .
$$

Integrating over $(\eta, 1)$, we have

$$
u^{\prime}(1) w^{\prime}(1)+2(n-1) \int_{\eta}^{1} \frac{1}{r} u^{\prime} w^{\prime} d r-f(u(\eta)) w(\eta)=0 .
$$

In view of (2.22) and (2.23) all three terms in (2.24) are positive, a contradiction.

We are now ready to give an alternative proof of the uniqueness result of Smoller and Wasserman [12].

Theorem 2.3. Under the conditions (2.13) and (2.14) the problem

$$
\Delta u+f(u)=0 \text { for }|x|<1, \quad u=0 \text { on }|x|=1
$$

can have at most one positive solution.

Proof. We imbed the problem (2.25) into a family of problems

$$
\Delta u+\lambda f(u)=0 \text { for }|x|<1, u=0 \text { on }|x|=1
$$

with a positive parameter $\lambda$. Let $u_{0}$ be a positive solution of (2.25). Then $(\lambda=$ $\left.1, u=u_{0}\right)$ is a solution of (2.26). By Theorem 2.2 we can continue this solution for decreasing $\lambda$, and the solution curve can have no turns. Next we claim that this solution branch $u(r, \lambda)$ cannot lose its positivity when decreasing $\lambda$. Since any positive solution of (2.26) is a decreasing function of $r$, the only way the positivity can get lost is that for some $\lambda_{1}$ we have $u^{\prime}\left(1, \lambda_{1}\right)=0$ and then for $\lambda<\lambda_{1}$ the solution $u(r, \lambda)$ becomes negative near $r=1$. Then $u_{\lambda}\left(r, \lambda_{1}\right)$ would have to be positive near $r=1$. (By the definition of $\lambda_{1}$ it is clear that $u_{\lambda}\left(r, \lambda_{1}\right)$ cannot be negative on an interval containing $r=1$. If $u_{\lambda}\left(r, \lambda_{1}\right)$ failed to be positive in some interval containing $r=1$, we could find a sequence $r_{n} \rightarrow 1$, such that $u_{\lambda}\left(r_{n}, \lambda_{1}\right)=0$. Let $\mu_{n} \rightarrow 1$ be points of positive local maxima of $u_{\lambda}\left(r, \lambda_{1}\right)$, i.e. $u_{\lambda}\left(\mu_{n}, \lambda_{1}\right)>0$ and $u_{\lambda}^{\prime}\left(\mu_{n}, \lambda_{1}\right)=0$. We now evaluate (2.11) at $r=\mu_{n}$. The first term on the left is negative, the second one is zero, and the third term is positive and tending to zero. The right-hand side tends to $-f(0)>0$, a contradiction.) Then as before we see that $z(r) \equiv r u_{r}\left(r, \lambda_{1}\right)-2 \lambda_{1} u_{\lambda}\left(r, \lambda_{1}\right)$ is a nontrivial solution of the linearization of (2.26) at $\lambda=\lambda_{1}$, contradicting Theorem 2.2 (notice that $z(1)=0$, and $z(r)$ is negative near $r=1$ ).

Our branch of solutions cannot approach the point $\lambda=0, u=0$ (just multiply the equation (2.26) by $u$ and integrate). Clearly (2.26) does not admit a trivial solution for $\lambda>0$. So the only possibility left is that $u(r, \lambda) \rightarrow \infty$ as $\lambda$ tends to some $\lambda_{0} \geq 0$. Setting $V(r, \lambda)=\frac{u(r, \lambda)}{u(0, \lambda)}$ it is standard to show, using $(2.21)$, that as $\lambda \rightarrow \lambda_{0}, V(r, \lambda)$ approaches a nontrivial nonnegative solution of

$$
\Delta v+\lambda_{0} c v=0 \text { for }|x|<1, \quad v=0 \text { on }|x|=1 .
$$


It follows that $\lambda_{0} c=\lambda_{1}$, where $\lambda_{1}$ is the principal eigenvalue of the Laplacian on the unit ball, and there is only one curve going to infinity. Since all positive solutions of (2.26) lie on a single curve, which admits no turns, it follows that for any $\lambda$ this problem has at most one positive solution.

Remark. It follows from the proof that (2.26) can have positive solutions only when $c \lambda>\lambda_{1}$. For increasing $\lambda$ the solution branch $u(r, \lambda)$ may lose its positivity, as the following numerical example shows. In one dimension we solved the problem $(u=u(x))$

$$
u^{\prime \prime}+\lambda\left(u-1-e^{-u}\right)=0 \text { on }(0,1), u(0)=u(1)=0 .
$$

Here $\lambda_{1}=\pi^{2}$. Our computations indicate a positive solution for $\pi^{2}<\lambda<\bar{\lambda}$, with $\bar{\lambda} \approx 35$. As $\lambda \downarrow \pi^{2}$ the solution goes to infinity, becoming uniformly large. As $\lambda$ increases past the critical value $\bar{\lambda}$, the solution becomes negative near the end points $x=0$ and $x=1$, after which it preserves the same nodal structure, and quickly goes to infinity. (Incidentally, it is easy to calculate $\bar{\lambda}$ analytically. Denote $f(u)=\left(u-1-e^{-u}\right), F(u)=\int_{0}^{u} f(t) d t$. Multiplying (2.27) by $u^{\prime}$, and integrating over $(0,1 / 2)$, we compute $u(1 / 2)=\beta$, where $\beta$ is defined by $\int_{0}^{\beta} f(u) d u=0, \beta \approx$ 2.69 for this problem. Multiplying $(2.27)$ by $u^{\prime}$ and integrating, $\frac{1}{2} u^{\prime^{2}}+\lambda F(u)=0$, from which we easily conclude that $\bar{\lambda}=2\left(\int_{0}^{\beta} \frac{d u}{\sqrt{-F(u)}}\right)^{2}$.)

\section{ACKNOWLEDGEMENT}

I wish to thank L. Nirenberg for a useful discussion on Lemma 2.1, and E.N. Dancer for useful communications.

\section{REFERENCES}

1. M.G. Crandall and P.H. Rabinowitz, Bifurcation, perturbation of simple eigenvalues and linearized stability, Arch. Rational Mech. Anal. 52, 161-180 (1973). MR 49:5962

2. E.N. Dancer, On the structure of solutions of an equation in catalysis theory when a parameter is large, J. Differ. Equations 37, 404-437 (1980). MR 82b:35018

3. L.C. Evans, Partial Differential Equations, Berkeley Lecture Notes in Mathematics. Vol. 3 A\&B (1994).

4. R. Gardner and L.A. Peletier, The set of positive solutions of semilinear equations in large balls, Proc. Royal Soc. Edinburgh 104A, 53-72 (1986). MR 88e:35063

5. B. Gidas, W.-M. Ni and L. Nirenberg, Symmetry and related properties via the maximum principle, Commun. Math. Phys. 68, 209-243 (1979). MR 80h:35043

6. M. Holzmann and H. Kielhöfer, Uniqueness of global positive solution branches of nonlinear elliptic problems, Math. Ann. 300, 221-241 (1994). MR 95m:35068

7. P. Korman, Steady states and long time behavior of some convective reaction-diffusion equations, To appear in Funkcialaj Ekvacioj.

8. P. Korman, Y. Li and T. Ouyang, Exact multiplicity results for boundary-value problems with nonlinearities generalizing cubic, Proc. Royal Soc. Edinburgh Ser. A 126A, 599-616 (1996). CMP 96:14

9. P. Korman, Y. Li and T. Ouyang, An exact multiplicity result for a class of semilinear equations, To appear in Comm. in PDE.

10. C.S. Lin and W.-M. Ni, A counterexample to the nodal domain conjecture and a related semilinear equation, Proc. Amer. Math. Soc. 102, 271-277 (1988). MR 88k:35014 
11. L.A. Peletier and J. Serrin, Uniqueness of positive solutions of semilinear equations in $R^{n}$, Arch. Rat. Mech. Anal. 81, 181-197 (1983). MR 84b:35046

12. J.A. Smoller and A.G. Wasserman, Existence, uniqueness, and nondegeneracy of positive solution of semilinear elliptic equations, Commun. Math. Phys. 95, 129-159 (1984). MR 86c:35058

Department of Mathematical Sciences, University of Cincinnati, Cincinnati, Ohio 45221-0025

E-mail address: korman@ucbeh.san.uc.edu 